\title{
Fluid shear stress changes cell morphology and regulates the expression of ATP6V1A and TCIRG1 mRNA in rat osteoclasts
}

\author{
DAWEI GUO ${ }^{1,2}$, QINGHONG ZHANG ${ }^{3}$, JINGWEN LI $^{1}$, XING LIANG ${ }^{2}$, \\ YUE CHEN ${ }^{2}$, HANG WANG ${ }^{2}$ and BO MENG ${ }^{1}$
}

\author{
${ }^{1}$ State Key Laboratory of Oral Diseases, West China College of Stomatology, and ${ }^{2}$ Department of Prosthodontics, \\ West China Hospital of Stomatology, Sichuan University, Chengdu 610041; ${ }^{3}$ Department of Prosthodontics, \\ The Affiliated Stomatology Hospital, Zhejiang University, Hangzhou 310006, P.R. China
}

Received August 3, 2009; Accepted September 7, 2009

DOI: $10.3892 / \mathrm{mmr} 00000236$

\begin{abstract}
Fluid shear stress (FSS) is a potent physical cell signal in the regulation of bone remodeling. Although the effects of FSS on bone cells of the osteoblastic lineage have been studied extensively, less is known about the direct effect of FSS on osteoclasts. In this study, we investigated the direct influence of FSS on rat osteoclasts isolated by a classic mechanical anatomical technique. Osteoclasts were exposed to an FSS of 2.9 dynes $/ \mathrm{cm}^{2}$ for a given period, or subjected to various magnitudes of FSS for $30 \mathrm{~min}$. Cell morphology under FSS was observed and the expression of the mRNA of ATP6V1A and TCIRG1, two crucial subunits of the vacuolar $\mathrm{H}^{+}$-ATPase gene, was analyzed by quantitative real-time PCR. Changes in osteoclast morphology were apparent after the application of FSS, including increased cell volume, strengthened refraction and decreased transmittance. Time-dependent and dose-dependent increases in ATP6V1A and TCIRG1 mRNA expression were seen in response to FSS within $30 \mathrm{~min}$. However, a duration of FSS exceeding $30 \mathrm{~min}$ induced a significant decrease in ATP6V1A and TCIRG1 mRNA expression. FSS therefore appears to be a potent stimulus that is sensed by rat osteoclasts and results in significant changes in cell morphology and the gene regulation of ATP6V1A and TCIRG1. These effects might be strictly dependent on the duration of FSS.
\end{abstract}

\section{Introduction}

Maintenance of appropriate bone mass requires the coordination of bone resorption by osteoclasts and bone formation by osteoblasts. It is well known that mechanical loading controls

Correspondence to: Dr Xing Liang, Department of Prosthodontics, West China Hospital of Stomatology, Sichuan University, Chengdu 610041, P.R. China

E-mail address: gdavy2007@hotmail.com

Key words: fluid shear stress, osteoclasts, vacuolar ATPase, ATP6V1A, TCIRG1 this cell coupling (1), and that mechanical loads applied to bone tissue result in the deformation of skeletal tissue, which induces the pressurization of interstitial fluid and stimulates its movement along pressure gradients (2). Within the confined geometry of lacunar-canalicular and haversian systems, interstitial fluid flow imparts shear stresses upon cell membranes (3). In vitro studies have indicated that the predicted levels of shear stress are potent regulators of bone cell behavior (4).

The mechanisms of flow-induced remodeling have been partially elucidated by in vitro studies that subjected osteoblasts or osteocytes to fluid shear stress (FSS). It is generally accepted that osteocytes are the primary mechanosensory cells in bone and elicit different biomechanical responses, such as the release of a number of signaling molecules; these in turn modulate the activities of osteoblasts and osteoclasts (5-9). Furthermore, increasing data suggest that not only osteocytes, but also osteoblasts and osteoprogenitors, are responsible for perceiving and responding to fluid flow (10-14). For example, FSS has been shown to modulate the key factors expressed in the osteoblasts and stromal cells that control osteoclast recruitment $(10,11)$. Osteoclasts, which reside in the bone marrow, are also possibly exposed to flow-induced shear stress. However, less is known about the direct effects of FSS on osteoclasts or the potential for osteoclasts to perceive and respond to fluid flow.

Osteoclasts are multinucleated giant cells that originate from hematopoietic precursor cells of the monocytic/ macrophage lineage. Under normal conditions, osteoclasts are the only cells that function to resorb the mineralized bone matrix (15). This function is carried out by the active secretion of protons into the resorption lacuna through the activity of a specialized osteoclastic vacuolar $\mathrm{H}^{+}$-ATPase (V-ATPase) present on the ruffled border membranes $(15,16)$. Suppression of the gene expression of V-ATPase by antisense RNA and DNA molecules inhibits bone resorption (17), and V-ATPase-specific inhibitors reduce bone resorption in vitro (18) and in vivo (19). Thus, V-ATPase plays an essential role in osteoclastic function.

$\mathrm{V}$-ATPase is composed of a peripheral $\mathrm{V}_{1}$ domain responsible for ATP hydrolysis and an integral $V_{0}$ domain responsible for proton translocation $(20,21)$. Subunit A, a 
protein located in the $\mathrm{V}_{1}$ domain, is the catalytic nucleotide binding site of V-ATPase, and the knockdown of its gene (ATP6V1A) impairs acid secretion (22). Subunit 'a' is an integral membrane protein of $\mathrm{V}_{0}$. Presently, four different isoforms of subunit ' $a$ ' have been identified, both in mice and humans, designated as a1, a2, a3 and a4. Among the four ' $a$ ' isoforms in mammals, a3 is a form that is enriched in osteoclasts and plays an essential role in bone resorption $(20,21)$. Disruption of the mouse or human a3 gene (TCIRG1) results in severe osteopetrosis $(23,24)$. Both subunit A (ATP6V1A) and a3 (TCIRG1) are highly expressed in osteoclasts, indicating their crucial roles in the resorption activity of the osteoclast.

Many in vitro studies have reported that FSS may regulate osteoclast differentiation in a co-culture system of osteoblasts and osteoclast precursors. This effect has been attributed to the modulation of key factors expressed in osteoblasts $(10,11)$, while few studies have considered the potential direct effects of FSS on osteoclasts, particularly on V-ATPase activity. In this study, we developed a parallel flow chamber to investigate the effect of FSS on the morphology of cultured rat osteoclasts, and examined the mRNA expression of ATP6V1A and TCIRG1 in response to FSS.

\section{Materials and methods}

Cell isolation and culture. Rat osteoclasts were isolated and cultured essentially as previously described (25). All procedures involving animal treatment were approved by the Council on Animal Care of Sichuan University. Briefly, 6-week-old male Sprague-Dawley rats (Western China Animal Center, P.R. China) were sacrificed by cervical dislocation. The tibiae and femura were removed and dissected free from adhering soft tissues under aseptic conditions. Epiphyseal heads were removed and the bones were rapidly cut into pieces with surgical scissors in a small beaker containing $30 \mathrm{ml}$ of $\alpha$-MEM (Hyclone, USA) culture medium at $4^{\circ} \mathrm{C}$. The beaker was vibrated on a mixer, then the supernatant was centrifuged and the resultant pellets were diluted to $10^{9} / \mathrm{ml}$ using $\alpha$-MEM culture medium containing 20\% fetal calf serum (Hyclone) and $100 \mathrm{U} / \mathrm{ml}$ penicillin and streptomycin (North China Pharmaceutical Corporation, P.R. China). The cell suspension was inoculated onto a glass slide in a petri dish or into 24-well culture plates, with or without dentine slices, and incubated at $37^{\circ} \mathrm{C}$ in $5 \% \mathrm{CO}_{2}$ in a humified tissue culture incubator. After $30 \mathrm{~min}$, the culture medium was replaced to remove nonadherent cells, and then changed three times at 3-h intervals.

Tartrate-resistant acid phosphatase staining. The cells cultured for a given period were washed with phosphate buffer solution (PBS) and fixed with glutaraldehyde for $10 \mathrm{~min}$. They were then rinsed with distilled water and air-dried. TRAP staining fluid (Sigma, USA) was added and the plates were incubated at $37^{\circ} \mathrm{C}$ for an additional $60 \mathrm{~min}$. After the removal of the Tartrate-resistant acid phosphatase (TRAP) solution, the plates were washed three times with distilled water and air-dried. The slices were sealed and observed. TRAP-positive cells with $\geq 3$ nuclei were considered to be osteoclasts.

Bone resorption assay. The cells were cultured together with dentine slices for three days, then the dentine slices were removed and cleaned ultrasonically for $60 \mathrm{~min}$. The slices were then washed with PBS, fixed in glutaraldehyde for 10 min, dehydrated in a graded alcohol series and incubated with $1 \%$ toluidine blue for 3-4 min. The slices were washed with distilled water and the resorption pits were observed by light microscopy (Olympus, Tokyo, Japan).

Fluid shear stress experiments. A parallel plate flow chamber was used to impose an FSS upon osteoclasts as previously described (14,25). The device was essentially a uniform rectangular parallel plate flow chamber and a liquid irrigation system. A glass slide was mounted in the flow chamber by sandwiching a silicone gasket between the glass slide and the chamber base. The liquid irrigation system contained an inlet and outlet so that fluid could perfuse the cells. Initiation of fluid flow generated laminar shear stress due to hydrostatic pressure between two storage pools, where the flow rate could be adjusted to obtain a specific shear stress level based on the Poiseuille law equation detailed in a previous study (25). The entire apparatus was maintained at $37^{\circ} \mathrm{C}$ and the medium was aerated with $95 \%$ air $/ 5 \% \mathrm{CO}_{2}$ during the experiments. All stress experiments were conducted under sterile conditions.

To investigate the effects of various stress intensities over a fixed time period (30 min), experimental groups were subjected to the following flow stress: 0 (control), 0.9, 2.9, 8.7 and 26.3 dynes $/ \mathrm{cm}^{2}$. In time-course studies, the cells were subjected to an FSS of 2.9 dynes $/ \mathrm{cm}^{2}$ for a given period. Polylysine (Sigma) was used to enhance the adherence of the cells to the glass surface. A flow of $\alpha$-MEM culture medium was used to impose FSS on the osteoclasts. The exposure time and range of stress intensities were chosen to ensure the maintenance of cell-substrate adhesion while keeping the cytoskeleton intact.

To observe the effects of FSS on the configuration of osteoclasts, the flow chamber was set up on the scanning stage of the microscope. During the process of stress loading, a clear field of view of the cell creeping slice $(\sim 10 \mathrm{~mm}$ in diameter) was selected at random and captured under a microscope. Image-Pro Plus analytical software (Media Cybernetics Corp., USA) was used to measure the cell area at each level of applied shear stress.

Quantitative real-time PCR ( $q P C R$ ). Following flow experiments, flow and static control samples were removed from the parallel plate flow chambers and cultured for an additional 2 h. Subsequently, medium was removed and, after washing with PBS, total RNA was extracted with Trizol Reagent (Sigma) following the manufacturer's instructions. The purity and quantity of RNA was determined spectrophotometrically and the integrity of the RNA preparations was examined by gel electrophoresis. Total RNA $(2 \mu \mathrm{g})$ was reverse transcribed into single-stranded cDNA by AMV reverse transcriptase and oligo(dT) primer (Sigma) following the company's recommended protocol. Amplification reactions were performed in $30 \mu \mathrm{l} \mathrm{PCR}$ buffer containing $10 \mu \mathrm{M}$ primers and $25 \mathrm{mM}$ dNTPs, as well as $5 \mathrm{U}$ Taq polymerase (Sigma) with $10 \mu \mathrm{M}$ labeled probes. Aliquots of cDNA were diluted 10 to 10,000 fold to generate relative standard curves, to which sample cDNA was compared. Sequences for the primers and probes are shown in Table I. Temperature cycling was as follows: initial denaturation at $94^{\circ} \mathrm{C}$ for $2 \mathrm{~min}$; denaturation at $94^{\circ} \mathrm{C}$ for $20 \mathrm{sec}$, 
Table I. Primer and probe sequences for qPCR.

Gene

Primer and probe sequences

\section{ATP6V1A}

Forward

5'-TAGGAGCAGTTTCTCCACCT-3'

Reverse

5'-CTTATCCAAGCCCCAGAACA-3'

Taqman probe

5'-CTGATCCAGTCACTTCTGCAAC-3'

\section{TCIRG1}

Forward

Reverse

5'-CTCAACTCCTTCAAGATGAAGAT-3'

Taqman probe

5'-CTTATCCAAGCCCCAGAACA-3'

GAPDH

Forward

5'-CACCCCAAAGGCCATGTGC-3'

Reverse

5'-TGGGTGTGAACCACGAGAA-3'

Taqman probe 5'-CTGCACCACAACTGCTTAGC-3'

A

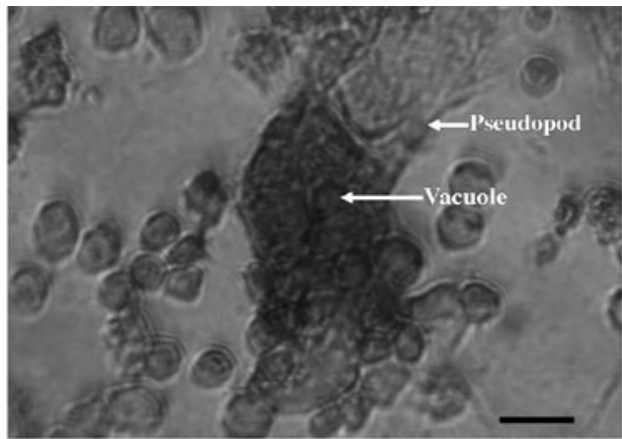

B

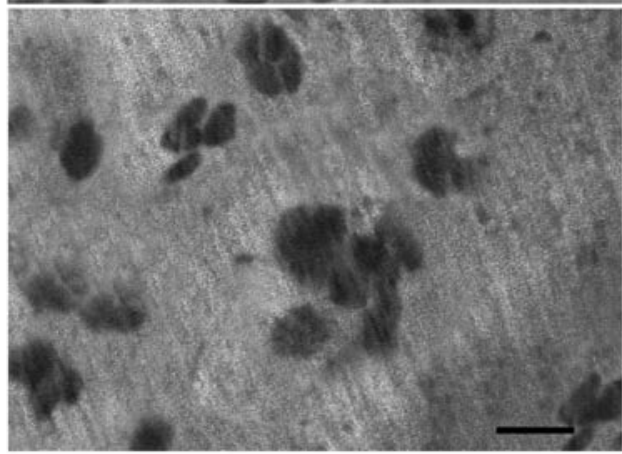

C

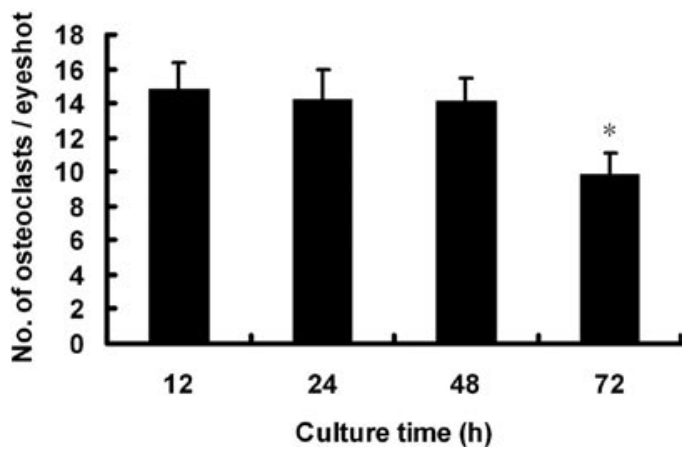

Figure 1. (A) Light microscopic image of a rat osteoclast stained for TRAP. The cell was larger than surrounding cells and had an irregular configuration. Spherical nuclei were dispersed throughout the cytoplasm. Some vesicles and pseudopodia were visible. Magnification x400; scale bar $20 \mu \mathrm{m}$. (B) Light microscopic photograph of a dentine slice cultured with marrow cells for 3 days. Irregularly shaped pits were clearly apparent. Magnification x400; scale bar $20 \mu \mathrm{m}$. (C) The number of osteoclasts was counted at various culture times. Results are shown as the mean \pm SD $(n=6)$. ${ }^{*} \mathrm{P}<0.05$ primer annealing at $55^{\circ} \mathrm{C}$ for $30 \mathrm{sec}$ and extension at $60^{\circ} \mathrm{C}$ for $40 \mathrm{sec}$ for 45 cycles. The relative target mRNA expression was computed from target cycle threshold $(\mathrm{Ct})$ values and GAPDH $\mathrm{Ct}$ values using the standard curve method.

Statistical analysis. Data are expressed as the mean \pm standard deviation (SD). Analysis of variance (ANOVA) followed by the Student's t-test (SPSS 12.0, Chicago, USA) were used for statistical analysis. At least four independent samples were examined for each experiment. A P-value $<0.05$ was considered statistically significant.

\section{Results}

Osteoclast identification. Cells isolated by osteoclast identification yielded a number of TRAP-positive multinucleated cells scattered among the monocytes. These displayed various morphologies (spherical, elliptical, kettle-shaped or irregular). Spherical nuclei were dispersed throughout the cytoplasm, and some vesicles and pseudopodia were occasionally visible (Fig. 1A). The cells were functional, as they produced resorption lacunae typical of osteoclasts when cultured on dentine slices (Fig. 1B). The number of osteoclasts was maintained at a relatively stable level over a culture period of $12-48 \mathrm{~h}$, but osteoclast number clearly decreased after $>72 \mathrm{~h}$ of culture (Fig. 1C).

Effects of FSS duration and magnitude on osteoclast morphology. As shown in Fig. 2A, significant changes in cell morphology, particularly in cell area, occurred when FSS was imposed on osteoclasts. Although some fluctuation was observed, there was a general increase in osteoclast area as the duration of FSS was extended (Fig. 2B). This area response was also dose-dependent, as the imposition of step-wise increases in FSS above 0.9 dynes $/ \mathrm{cm}^{2}$ resulted in a step-wise change in osteoclast area (Fig. 2C).

Effect of FSS duration on the expression of ATP6V1A and TCIRG1 mRNA. FSS applied for 15 min clearly increased the expression of ATP6V1A and TCIRG1 mRNA compared to the levels seen in control unstressed cells. The levels of ATP6V1A and TCIRG1 mRNA expression increased to a peak at $30 \mathrm{~min}$. However, extending the duration of FSS to 60 min induced a significant decrease in the expression of ATP6V1A and TCIRG1 mRNA compared to the unstressed controls. Further FSS exposure led to further down-regulation of mRNA expression (Fig. 3).

Effect of FSS magnitude on the expression of ATP6VIA and TCIRG1 mRNA. As shown in Fig. 4, application of FSS at 0.9 dynes $/ \mathrm{cm}^{2}$ for $30 \mathrm{~min}$ significantly increased the expression of ATP6V1A and TCIRG1 mRNA compared to the unstressed controls. As the magnitude of FSS was increased, the levels of ATP6V1A and TCIRG1 mRNA were up-regulated proportionally.

\section{Discussion}

It is difficult to study osteoclasts in vitro since they are relatively scarce, terminally differentiated, adherent to 
A
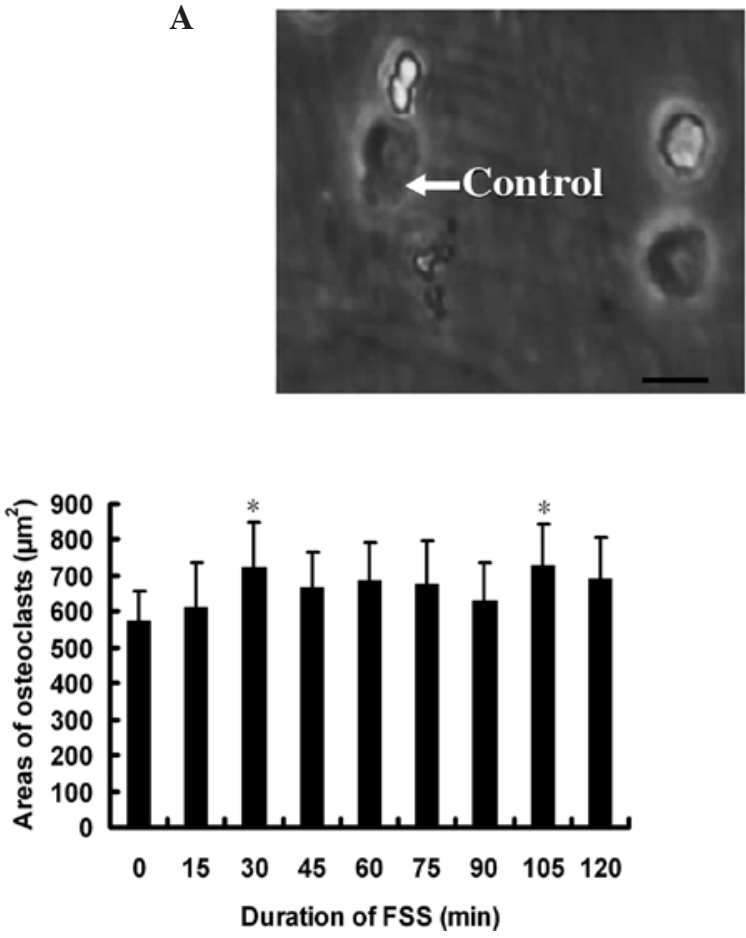
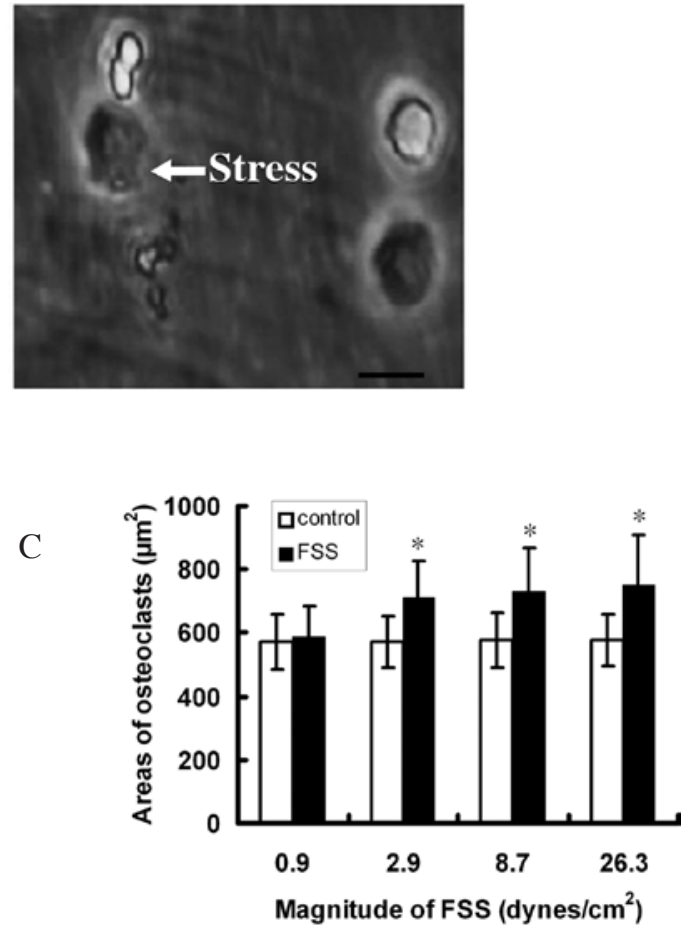

Figure 2. Effects of fluid shear stress (FSS) on cultured rat osteoclast morphology. (A) Light microscopic photographs of osteoclasts before stress (left) and following fluid shear stress for $30 \mathrm{~min}$ (right). Magnification x400; scale bar $20 \mu \mathrm{m}$. (B) Changes in osteoclast area calculated using Image-Pro Plus analytical software under static conditions or following an FSS of 2.9 dynes/ $\mathrm{cm}^{2}$ for the indicated times. (C) Changes in osteoclast area under static conditions or after being subjected to the indicated FSS stress intensity $\left(\right.$ dynes $\left./ \mathrm{cm}^{2}\right)$ for $30 \mathrm{~min}$. Results are shown as the mean $\pm \mathrm{SD}(\mathrm{n}=6)$. * $\mathrm{P}<0.05$.

A

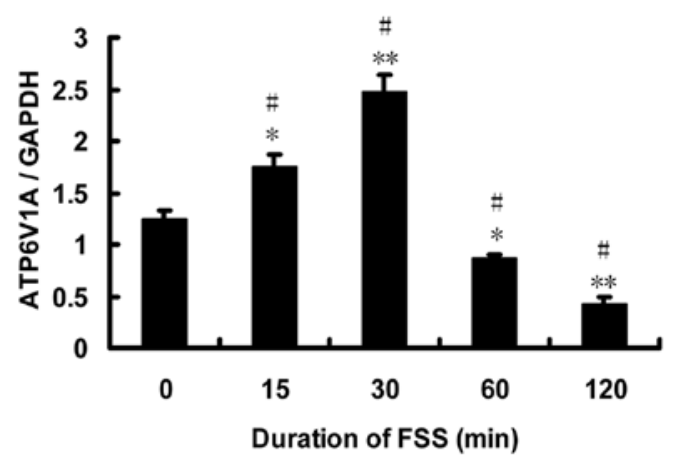

B

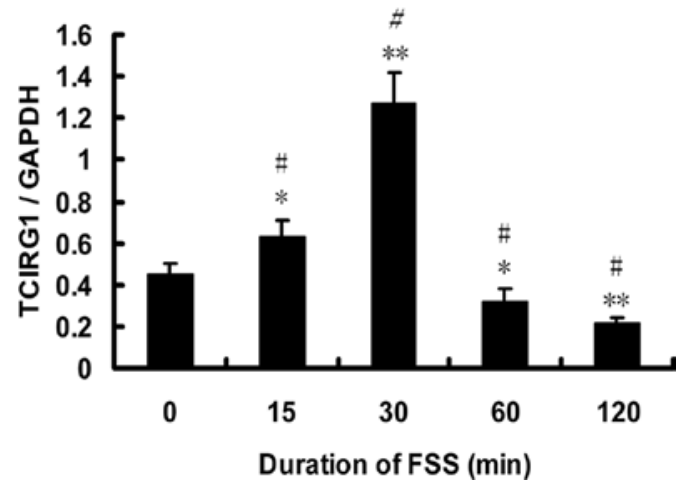

Figure 3. Effect of FSS duration on the expression of (A) ATP6V1A and (B) TCIRG1 mRNA in rat osteoclasts. After a 24-h culture, the osteoclasts were subjected to an FSS of 2.9 dynes $/ \mathrm{cm}^{2}$ for 15, 30, 60 and $120 \mathrm{~min}$. Expression of ATP6V1A and TCIRG1 mRNA was measured by qPCR, and the data were normalized to GAPDH expression. Results are shown as the mean \pm SD $(\mathrm{n}=4) .{ }^{*} \mathrm{P}<0.05 ;{ }^{* * *} \mathrm{P}<0.01$, compared with the 0 -min (control) group. ${ }^{\#} \mathrm{P}<0.05$, compared with the other experimental groups.

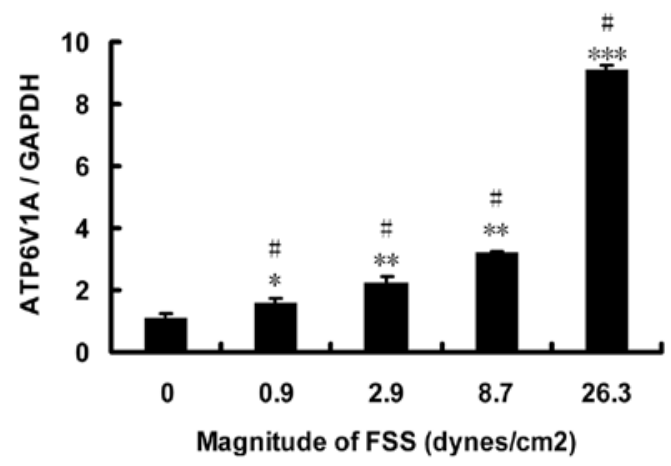

B

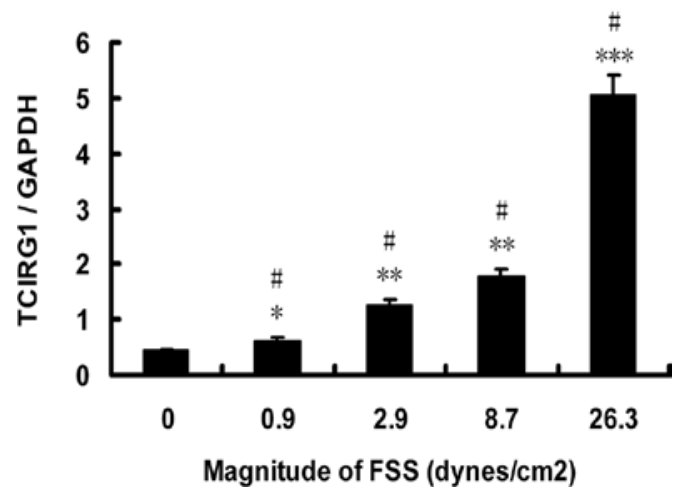

Figure 4. Effect of FSS magnitude on the expression of (A) ATP6V1A and (B) TCIRG1 mRNA in rat osteoclasts. After a 24-h culture, the osteoclasts were subjected to an FSS of $0.9,2.9,8.7$ and 26.3 dynes $/ \mathrm{cm}^{2}$ for $30 \mathrm{~min}$. Expression of ATP6V1A and TCIRG1 mRNA was measured by qPCR, and the data were normalized to GAPDH expression. Results are shown as the mean $\pm \mathrm{SD}(\mathrm{n}=4)$. ${ }^{*} \mathrm{P}<0.05 ;{ }^{* *} \mathrm{P}<0.01 ;{ }^{* * * *} \mathrm{P}<0.001$, compared with the 0 -min (control) group. ${ }^{*} \mathrm{P}<0.05$, compared with the other experimental groups. 
mineralized surfaces and fragile (26). To date, no ideal methods have been established to provide large populations of highly purified and activated osteoclasts. In this study, we used a classic mechanical anatomical technique to obtain osteoclasts derived directly from animal bone marrow tissue. These cells are most likely to have characteristics closely resembling those of osteoclasts in physiological conditions. In addition, the cells obtained by this procedure were sufficient in number for the experiments and, more importantly, the numbers were maintained at a relatively stable level over a period of 12-48 h. All the experiments described in the present study were completed within $24 \mathrm{~h}$.

Changes in osteoclast morphology were apparent after the application of an FSS. This was particularly noticable as increases in osteoclast volume (Fig. 2), which were confirmed by strengthened refraction and decreased transmittance following FSS. These changes in cell morphology may be related to inner alterations of the osteoclast. It has become clear that osteoblasts or osteocytes exposed to fluid flow elicit a number of biomechanical responses, such as the activation of intracellular signaling cascades including mitogen-activated protein kinases (MAPK), PKA and PKC pathways $(10,14,27)$, the release of soluble factors including ATP, nitric oxide (NO) and prostaglandin $\mathrm{E}_{2}\left(\mathrm{PGE}_{2}\right)(6,28,29)$, cytoskeleton reorganization (30), and the regulation of bone-specific marker expression including Cbfa1/Runx2, osteopontin, cyclooxygenase-2 (Cox-2) and several extracellular matrix proteins $(14,31,32)$. We therefore speculated that osteoclasts are also capable of perceiving fluid flow and respond to FSS by the activation of intracellular signaling cascades and subsequent changes in gene regulation, which gave rise to the observed morphological changes. The application of FSS for $30 \mathrm{~min}$ resulted in a dose-dependent increase in the mRNA levels of ATP6V1A and TCIRG1, as compared to the unstressed controls (Fig. 3). V-ATPases play a critical role in the resorption activity of osteoclasts, and V-ATPase expression, in particular of ATP6V1A and TCIRG1, increases with the activity of osteoclasts $(15,16)$. Therefore, it is likely that FSS also increased the activity of the osteoclasts in the present study. However, this activation is apparently time-dependent, occurring within the first 30 min of stress; extending the duration of the FSS beyond 30 min resulted in a loss of expression of ATP6V1A and TCIRG1 mRNA. Prolonged FSS may therefore have an inhibitory effect on V-ATPase expression.

These results differ from those reported in other studies using osteoblasts or other bone cells. Many in vitro studies in co-cultures of osteoblasts and osteoclasts indicate that mechanical stress induces the activation and differentiation of osteoblasts, while inhibiting osteoclast differentiation $(7,8,11,13,29)$. For example, Kim et al reported that physiological levels of loading-induced fluid flow decreased osteoclast formation in a co-culture system of marrow stromal cells and osteoclast precursors by decreasing the RANKL/OPG mRNA ratio (11). Furthermore, Suzuki et al suggested that mechanical stress directly suppresses osteoclast differentiation in RAW264.7 cells (33). Taken together, these studies consistently suggest that mechanical load is capable of depressing osteoclastogenesis and inhibiting bone resorption.

On the other hand, there are studies indicating that mechanical stress regulates the key factors expressed in osteo- blasts and stromal cells, and induces osteoclast differentiation. Judex et al reported that RANKL mRNA was increased in the murine tibia after exposing mice to vibrational stress (34). Zhu et al found that the RANKL/OPG ratio was increased during mechanically-induced bone formation by distraction osteogenesis in the mandibula of rats (35). Mehrotra et al indicated that fluid flow increased RANKL expression in primary murine calvarial osteoblasts (10). These data suggest that mechanical loading could enhance osteoclastogenesis.

The contradictory results in the literature may partly be due to the use of different mechanical conditions. A study by Kreja et al demonstrated that in human primary osteoblastic cells, intermittent stretching increased RANKL expression, whereas continuous loading had no effect, regardless of the strain magnitude or the duration of loading (36). In addition, in vitro studies have suggested that altering the flow rate at a fixed fluid shear stress alters the ability of bone cells to respond to fluid flow $(13,37)$. Based on these studies, we deduced that different types and frequencies of stress would have varying effects on bone cells. The present study confirms that different durations of stress induce different osteoclast reactions.

In the current study, a prolonged duration of stress gradually became inhibitory to the expression of ATP6V1A and TCIRG1 mRNA. This may be associated with several cellular events induced by the FSS. Cells isolated from animal bone marrow include not only osteoclasts, but also osteoblasts, macrophages and others (26). In the current experiments, these cells were simultaneously exposed to flow fluid. Many in vitro studies have indicated that osteoblasts subjected to FSS release a number of autocrine/paracrine factors, such as ATP, $\mathrm{NO}$ and $\mathrm{PGE}_{2},(6,28,29)$. Furthermore, McAllister et al also observed the release of $\mathrm{NO}$ and prostaglandin following the exposure of osteoclast-like cells to FSS (38). It is known that $\mathrm{NO}$ is antiapoptotic and contributes to osteoblast activation $(39,40) . \mathrm{PGE}_{2}$ has been shown to enhance the anabolic effects of mechanical loading (41). Pharmacological inhibition of $\mathrm{PGE}_{2}$ or $\mathrm{NO}$ production in vivo inhibits bone formation after the addition of exogenous loads $(42,43)$. Moreover, the induction of prostaglandin synthesis in response to fluid flow appears to be dependent upon the release of ATP (28). These results indicate that these released factors can decrease the activity of osteoclasts. Taken together, we speculate that the signaling molecules released by osteoblasts or osteoclasts may be transmitted via the cytoskeleton, which is physically linked to ion channels, as well as the intracellular signal transduction pathways, thus regulating the expression of some genes in osteoclasts, such as ATP6V1A and TCIRG1.

In summary, FSS is a potent stimulus that is sensed by osteoclasts, resulting in significant changes in cell morphology and in the expression of ATP6V1A and TCIRG1 mRNA. These effects were strictly dependent on the duration of FSS; however, the mechanisms by which FSS activates osteoclasts and regulates the mRNA expression of ATP6V1A and TCIRG1 is in need of further investigation.

\section{Acknowledgements}

This work was supported by a Grant from the National Natural Science Foundation of China (No. 30672345). 


\section{References}

1. Robling AG, Castillo $\mathrm{AB}$ and Turner $\mathrm{CH}$ : Biomechanical and molecular regulation of bone remodeling. Ann Rev Biomed Eng 8: 455-498, 2006

2. Piekarski $\mathrm{K}$ and Munro M: Transport mechanism operating between blood supply and osteocytes in long bones. Nature 269 : 80-82, 1977.

3. Weinbaum S, Cowin SC and Zeng Y: A model for the excitation of osteocytes by mechanical loading-induced bone fluid shear stresses. J Biomech 27: 339-360, 1994.

4. Riddle RC and Donahue HJ: From streaming-potentials to shear stress: 25 years of bone cell mechanotransduction. J Orthop Res 27: 143-149, 2009

5. Bonewald LF and Johnson ML: Osteocytes, mechanosensing and Wnt signaling. Bone 42: 606-615, 2008.

6. McGarry JG, Klein-Nulend J and Prendergast PJ: The effect of cytoskeletal disruption on pulsatile fluid flow-induced nitric oxide and prostaglandin $\mathrm{E}_{2}$ release in osteocytes and osteoblasts. Biochem Biophys Res Commun 330: 341-348, 2005.

7. Tan SD, De Vries TJ, Kuijpers-Jagtman AM, Semeins CM, Everts V and Klein-Nulend J: Osteocytes subjected to fluid flow inhibit osteoclast formation and bone resorption. Bone 41: 745-751, 2007.

8. You L, Temiyasathit S, Lee P, et al: Osteocytes as mechanosensors in the inhibition of bone resorption due to mechanical loading. Bone 42: 172-179, 2008.

9. Santos A, Bakker AD, Zandieh-Doulabi B, Semeins CM and Klein-Nulend J: Pulsating fluid flow modulates gene expression of proteins involved in Wnt signaling pathways in osteocytes. J Orthop Res Apr 7, 2009 E-pub ahead of print.

10. Mehrotra M, Saegusa M, Wadhwa S, Voznesensky O, Peterson D and Pilbeam C: Fluid flow induces Rankl expression in primary murine calvarial osteoblasts. J Cell Biochem 98: 1271-1283, 2006.

11. Kim CH, You L, Yellowley CE and Jacobs CR: Oscillatory fluid flow-induced shear stress decreases osteoclastogenesis through RANKL and OPG signaling. Bone 39: 1043-1047, 2006.

12. Lee DY, Yeh CR, Chang SF, et al: Integrin-mediated expression of bone formation-related genes in osteoblast-like cells in response to fluid shear stress: roles of extracellular matrix, Shc and mitogen-activated protein kinase. J Bone Miner Res 23: 1140-1149, 2008.

13. Riddle RC, Hippe KR and Donahue HJ: Chemotransport contributes to the effect of oscillatory fluid flow on human bone marrow stromal cell proliferation. J Orthop Res 26: 918-924, 2008.

14. Grellier M, Bareille R, Bourget C and Amédée J: Responsiveness of human bone marrow stromal cells to shear stress. J Tissue Eng Regen Med 3: 302-309, 2009.

15. Bar-Shavit Z: The osteoclast: a multinucleated, hematopoieticorigin, bone-resorbing osteoimmune cell. J Cell Biochem 102: 1130-1139, 2007.

16. Henriksen K, Sørensen MG, Jensen VK, Dziegiel MH, Nosjean $\mathrm{O}$ and Karsdal MA: Ion transporters involved in acidification of the resorption lacuna in osteoclasts. Calcif Tissue Int 83: 230-242, 2008.

17. Laitala $\mathrm{T}$ and Vaananen HK: Inhibition of bone resorption in vitro by antisense RNA and DNA molecules targeted against carbonic anhydrase II or two subunits of vacuolar $\mathrm{H}(+)$-ATPase. J Clin Invest 93: 2311-2318, 1994.

18. Sørensen MG, Henriksen K, Neutzsky-Wulff AV, Dziegiel MH and Karsdal MA: Diphyllin, a novel and naturally potent V-ATPase inhibitor, abrogates acidification of the osteoclastic resorption lacunae and bone resorption. J Bone Miner Res 22: 1640-1648, 2007

19. Niikura K: Comparative analysis of the effects of a novel vacuolar adenosine 5'-triphosphatase inhibitor, FR202126 and doxycycline on bone loss caused by experimental periodontitis in rats. J Periodontol 77: 1211-1216, 2006.

20. Yao G, Feng H, Cai Y, Qi W and Kong K: Characterization of vacuolar-ATPase and selective inhibition of vacuolar-H(+)ATPase in osteoclasts. Biochem Biophys Res Commun 357: 821-827, 2007.

21. Xiao YT, Xiang LX and Shao JZ: Vacuolar H(+)-ATPase. Int J Biochem Cell Biol 40: 2002-2006, 2008.
22. Horng JL, Lin LY, Huang CJ, Katoh F, Kaneko T and Hwang PP: Knockdown of V-ATPase subunit A (atp6v1a) impairs acid secretion and ion balance in zebrafish (Danio rerio). Am J Physiol Regul Integr Comp Physiol 292: 2068-2076, 2007.

23. Li YP, Chen W, Liang Y, Li E and Stashenko P: Atp6i-deficient mice exhibit severe osteopetrosis due to loss of osteoclast-mediated extracellular acidification. Nat Genet 23: 447-451, 1999.

24. Frattini A, Orchard PJ, Sobacchi C, et al: Defects in TCIRG1 subunit of the vacuolar proton pump are responsible for a subset of human autosomal recessive osteopetrosis. Nat Genet 25: 343-346, 2000.

25. Zhang Q, Liang X, Zhu B, Dong Q, et al: Effects of fluid shear stress on mRNA expression of carbonic anhydrase II in polarized rat osteoclasts. Cell Biol Int 30: 714-720, 2006.

26. Kartsogiannis V and Ng KW: Cell lines and primary cell cultures in the study of bone cell biology. Mol Cell Endocrinol 228: 79-102, 2004.

27. Liu D, Genetos DC, Shao Y, et al: Activation of extracellularsignal regulated kinase (ERK1/2) by fluid shear is $\mathrm{Ca}(2+)$ - and ATP-dependent in MC3T3-E1 osteoblasts. Bone 42: 644-652, 2008.

28. Genetos DC, Geist DJ, Liu D, Donahue HJ and Duncan RL: Fluid shear-induced ATP secretion mediates prostaglandin release in MC3T3-E1 osteoblasts. J Bone Miner Res 20: 41-49, 2005.

29. Riddle RC, Taylor AF, Rogers JR and Donahue HJ: ATP release mediates fluid flow-induced proliferation of human bone marrow stromal cells. J Bone Miner Res 22: 589-600, 2007.

30. Myers KA, Rattner JB, Shrive NG and Hart DA: Osteoblast-like cells and fluid flow: cytoskeleton-dependent shear sensitivity. Biochem Biophys Res Commun 364: 214-219, 2007.

31. Kreke MR, Huckle WR and Goldstein AS: Fluid flow stimulates expression of osteopontin and bone sialoprotein by bone marrow stromal cells in a temporally dependent manner. Bone 36: 1047-1055, 2005.

32. Wu CC, Li YS, Haga JH, et al: Roles of MAP kinases in the regulation of bone matrix gene expressions in human osteoblasts by oscillatory fluid flow. J Cell Biochem 98: 632-641, 2006.

33. Suzuki N, Yoshimura Y, Deyama Y, Suzuki K and Kitagawa Y: Mechanical stress directly suppresses osteoclast differentiation in RAW264.7 cells. Int J Mol Med 21: 291-296, 2008.

34. Judex S, Zhong N, Squire ME, et al: Mechanical modulation of molecular signals which regulate anabolic and catabolic activity in bone tissue. J Cell Biochem 94: 982-994, 2005.

35. Zhu WQ, Wang X, Wang XX and Wang ZY: Temporal and spatial expression of osteoprotegerin and receptor activator of nuclear factor-kappaB ligand during mandibular distraction in rats. $\mathrm{J}$ Craniomaxillofac Surg 35: 103-111, 2007.

36. Kreja L, Liedert A, Hasni S, Claes L and Ignatius A: Mechanical regulation of osteoclastic genes in human osteoblasts. Biochem Biophys Res Commun 368: 582-587, 2008.

37. Mullender MG, Dijcks SJ, Bacabac RG, Semeins CM, van Loon JJ and Klein-Nulend J: Release of nitric oxide, but not prostaglandin $\mathrm{E}_{2}$, by bone cells depends on fluid flow frequency. J Orthop Res 24: 1170-1177, 2006.

38. McAllister TN, Du T and Frangos JA: Fluid shear stress stimulates prostaglandin and nitric oxide release in bone marrowderived preosteoclast-like cells. Biochem Biophys Res Commun 270: 643-648, 2000.

39. Chang CC, Liao YS, Lin YL and Chen RM: Nitric oxide protects osteoblasts from oxidative stress-induced apoptotic insults via a mitochondria-dependent mechanism. J Orthop Res 24: 1917-1925, 2006.

40. Rahnert J, Fan X, Case N, et al: The role of nitric oxide in the mechanical repression of RANKL in bone stromal cells. Bone 43: 48-54, 2008.

41. Li J, Duncan RL, Burr DB, Gattone VH and Turner $\mathrm{CH}$ : Parathyroid hormone enhances mechanically induced bone formation, possibly involving L-type voltage-sensitive calcium channels. Endocrinology 144: 1226-1233, 2003.

42. Li J, Burr DB and Turner CH: Suppression of prostaglandin synthesis with NS-398 has different effects on endocortical and periosteal bone formation induced by mechanical loading. Calcif Tissue Int 70: 320-329, 2002.

43. Afzal F, Polak J and Buttery L: Endothelial nitric oxide synthase in the control of osteoblastic mineralizing activity and bone integrity. J Pathol 202: 503-510, 2004. 